\title{
The ideas of A.M. Baldin on overcoming the reductionism principle in relativistic physics
}

\author{
Anton A. Baldin ${ }^{1,2}$ \\ ${ }^{1}$ Joint Institute for Nuclear Research, Dubna, 141980 Russia \\ ${ }^{2}$ Institute for Advanced Studies "OMEGA", Dubna, 141980 Russia
}

\begin{abstract}
The reductionism principle and the role of the Standard Model in the general paradigm of modern physics are discussed. The structure of the laws of nature is considered based on the idea of symmetry. The criteria of applicability of variables used for description of relativistic nuclear collisions and the ideas of A. M. Baldin on the notion "elementary particle" are discussed. Particle production is considered using the main parameter of the Lobachevsky geometry, the angle of parallelism.
\end{abstract}

Reductionism is a philosophical idea regarding the associations between phenomena which can be described in terms of other simpler or more fundamental phenomena.

The principle of reductionism is one of the basic principles in modern physics, especially elementary particle physics.

A. M. Baldin was much interested in the structure of fundamental laws of nature, mentioning A. Einstein concerning the possibility of deducing all facts from the first principles (ideal theory). It is said in [1]: "It is impossible to deduce nuclear physics, including relativistic nuclear physics, from quantum chromodynamics without using auxiliary hypotheses needed to be checked experimentally. The verification of such hypotheses of a rather general nature is not less important than the verification of quantum chromodynamics. For instance, a detailed probe of the correlation depletion principles and the self-similarity is a task of paramount importance. ... However, both the Lagrangian properties and the self-similarity are the consequences of symmetry. Symmetry was considered by ancient Greek philosophers as a particular case of harmony i.e. the concordance of the parts within the whole" [1].

We give here the diagram (Fig. 1) of the physical laws and symmetries, as they were understood by A. M. Baldin [1]. 
A.M.Baldin

JINR Rapid Comm. №5, 1996

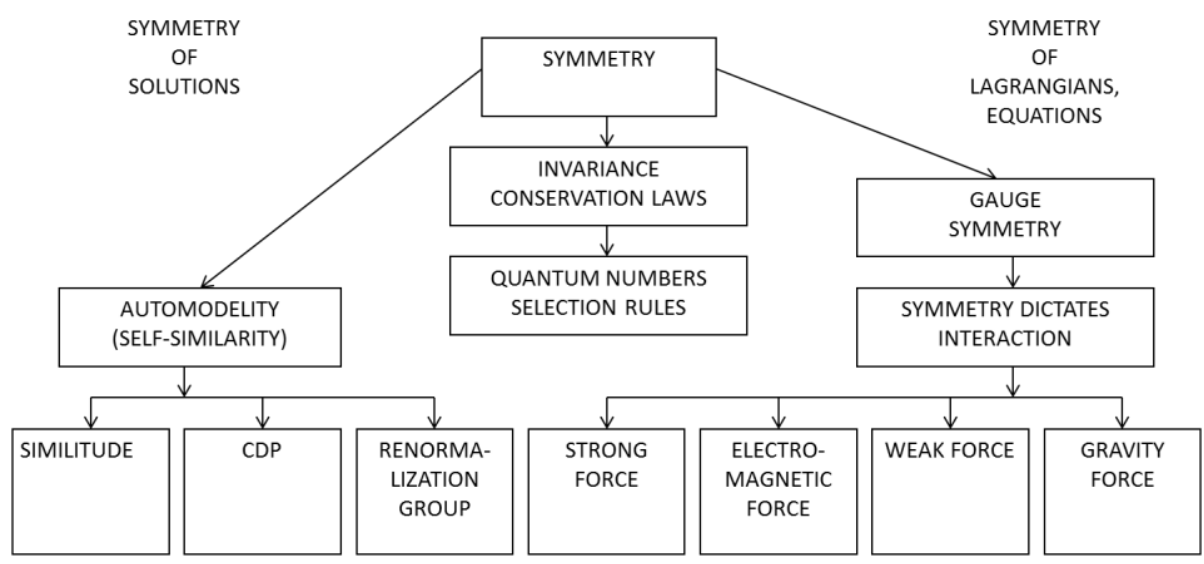

Schematic diagram illustrating the role of symmetry in fundamental physics

Figure 1. Symmetry includes symmetry of solutions and symmetry of equations [1]

Surely, the nature of elementary particles was of great interest, too [2].

It is not a mere accident that the internal structure of the proton was discovered by $\mathrm{R}$. Hofstadter [3], the parton model was developed by Feinman [4] and Bjorken [5], and the proton polarizability was discovered by A. M. Baldin [6] in a short time interval.

Considering the notion of elementary particle, A. Akhieser and M. Rekalo wrote: "It should be noted that it was assumed not long ago that an elementary particle cannot have a size and should necessarily be point-like. This opinion was related to the fact that a nonpoint-like particle was considered as a solid rigid sphere. As, for example, in the well known text book by Landau and Lifshitz... In reality, however, the existence of a particle size is not equivalent to its rigidity." [7].

A. M. Baldin arrived at the conclusion that the notion of an elementary particle itself depends on the statement of the problem. This is close to the idea put forward by W. Heisenberg [2]. For example, a helium atom can be treated as an elementary object in the theory of superconductivity and superfluidity, and the field theory can be applied to describe its behavior in these conditions. Another example that lies quite far from particle physics is that the motion of celestial bodies in many cases can be described as the motion of point objects possessing certain masses.

Following the observability principle formulated by E. Mach and W. Heisenberg stating that quantum mechanics should be founded on relationships between quantities which in principle are observable, A. M. Baldin introduced the notion of $b_{i k}$ for description of the character of interaction between elementary particles [8].

It is defined as follows:

$$
b_{i k}=-\left(U_{i}-U_{k}\right)^{2}=2\left[\left(U_{i} U_{k}\right)-1\right]=2\left[\frac{E_{i} E_{k}-\vec{p}_{i} \vec{p}_{k}}{m_{i} m_{k}}-1\right],
$$

where $U_{i}$ and $U_{k}$ are the four-velocities of particles $i$ and $k ; E_{i}$ and $E_{k}$ are their total energies; $\vec{p}_{i}, \vec{p}_{k}$ momenta, and $m_{i}, m_{k}$ masses of these particles. 


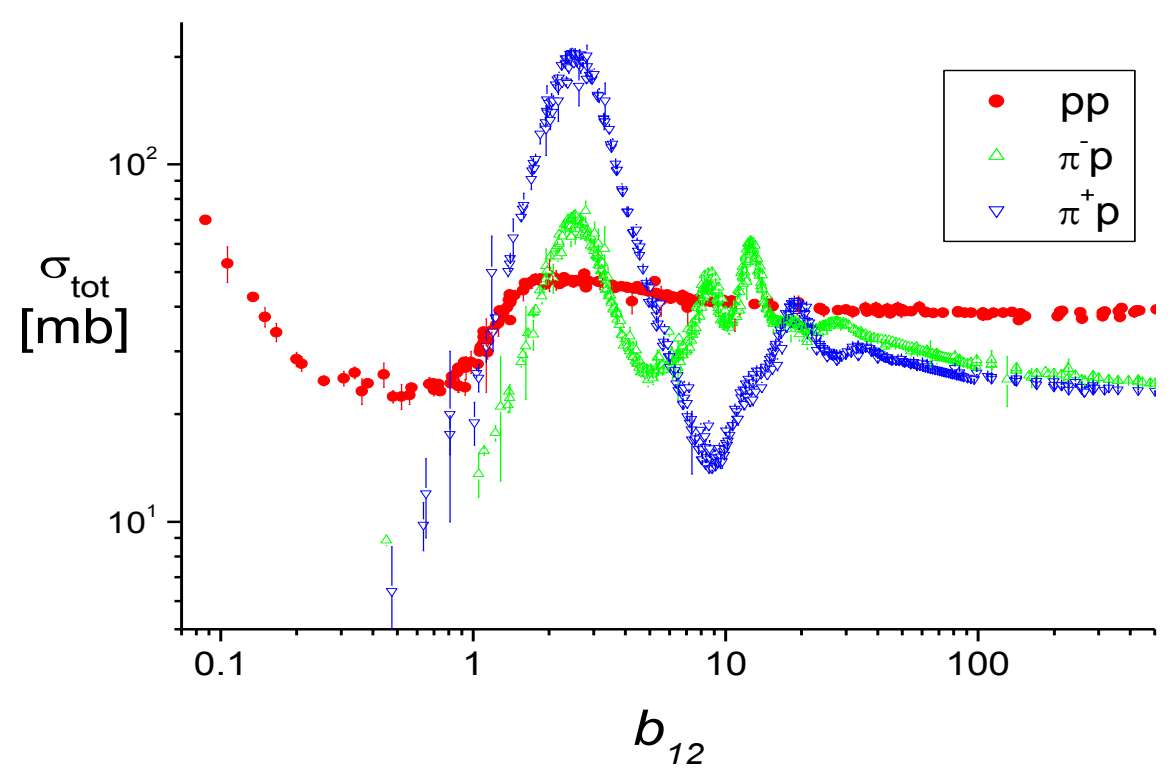

Figure 2. Total cross section of particle production as a function of $b_{i k}$. [9]

This variable, being the squared relative four momentum of two particles, is a relativistic invariant. Let us consider Lobachevsky space and introduce rapidity as the distance between two points in Lobachevsky space. In terms of rapidity, the variable $b_{i k}$ is written as follows:

$$
b_{i k}=2\left[\left(U_{i} U_{k}\right)-1\right]=2\left[\operatorname{ch} \rho_{i k}-1\right],
$$

where $\rho_{i k}$ is the relative rapidity of two particles $i$ and $k$ in Lobachevsky space.

Thus defined variable $b_{i k}$ can be applied for the following particle classification (Fig. 2):

(i) $0 \leq b_{i k} \leq 10^{-2}$ : relativistic nuclear physics, where nucleons can be considered as point objects;

(ii) $b_{i k} \sim 1$ : excitation of internal degrees of freedom of hadrons;

(iii) $b_{i k}>>1$ should, in principle, be described by quantum chromodynamics.

It should be noted, however, that a strong limitation for application of $b_{i k}$ is that this variable does not form a metric space.

In order to avoid the inconveniencies introduced by the variable $b_{i k}$ upon analysis of relativistic multiparticle systems, it is reasonable to consider variables forming the metric space, namely, the Lobachevsky space, i.e., relative rapidities $\rho_{i k}$ (Fig. 3) [10]. Relative rapidity of two particles $i$ and $k$ is defined as follows:

$$
\operatorname{ch} \rho_{i k}=U_{i} U_{k} \text {. }
$$




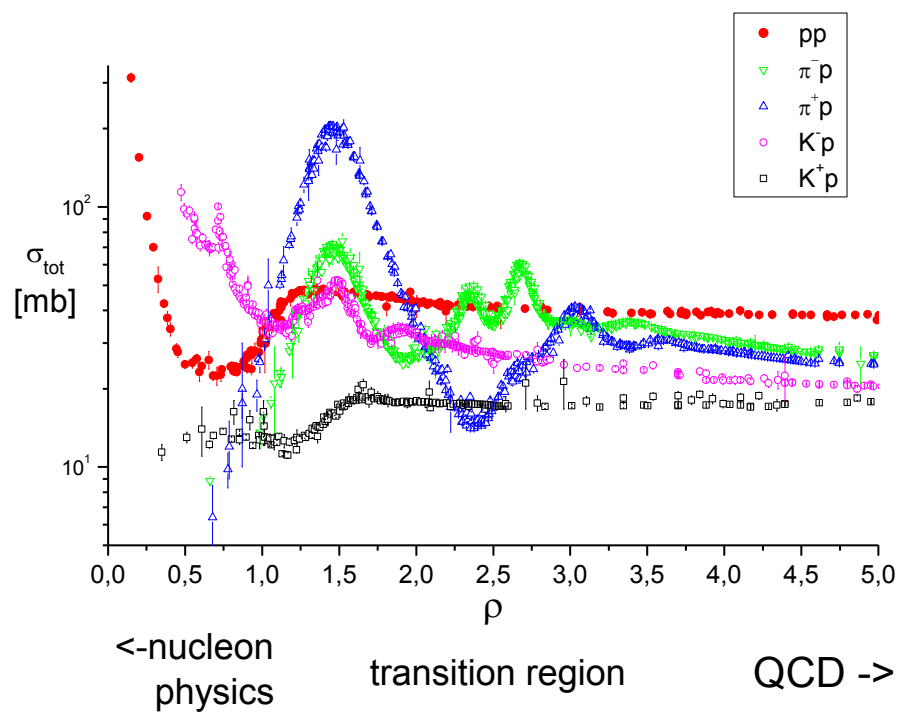

Figure 3. Total cross section of particle production as a function of $\rho_{i k}$. [11]

Lobachevsky geometry is widely used in relativistic physics [12-15]. It should be noted that it is not an accident. According to F. Klein, the modern relativity principle in physics amounts to the same thing as the Lorentz group theory, which is isomorphic to the group of isometries of the Lobachevsky space.

Let us speak in more detail about one of its basic concepts, the angle of parallelism, and give it a physical interpretation.

The variable characterizing the closeness of a produced particle to the absolute is based on the Lobachevsky angle of parallelism. According to the Lobachevsky geometry, any distance $h$ corresponds to the angle of parallelism,

$$
\Pi_{L}(h)=2 \operatorname{arctg}\left(e^{-h}\right) .
$$

The variable characterizing the closeness to the absolute for a triangle in the Lobachevsky rapidity space with three particles situated at its vertices is determined as (see Figs. 4, 5)

$$
\Delta_{12}^{3}=2 \Pi_{L}(h)-\alpha_{3} .
$$

It should be emphasized that this variable is determined from experimentally measurable quantities, particle angles, momenta, and masses, and is relativistically invariant.

As was noted in [14], the pronounced narrow maximum of particle production is observed in experimental distributions, which corresponds to the value of the Lobachevsky function $\Delta_{12}^{3}$ corresponding to the rapidity of colliding particles $\rho_{12}$, $\Pi_{L}\left(\rho_{12}\right)=2 \operatorname{arctg}\left(e^{-\rho_{12}}\right)$ (see Fig. 4). 


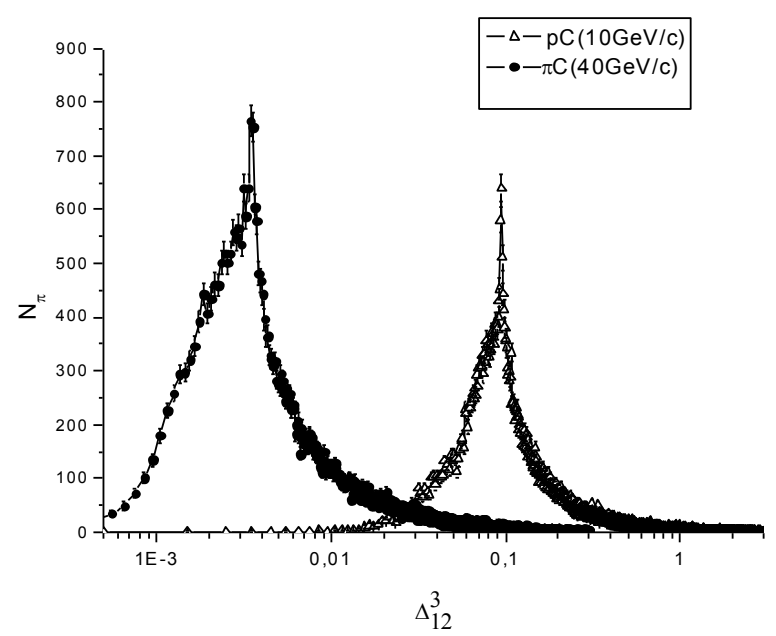

Fig. 4 Peaks in pion distributions for two reactions $p(10 \mathrm{GeV} / \mathrm{c})+\mathrm{C} \rightarrow \pi$ and $\pi^{-}(40 \mathrm{GeV} / \mathrm{c})+C \rightarrow \pi$

Fig. 5 shows pion distributions as functions of $\Delta_{12}^{3}$ for the experimental data obtained in $n+p$ interactions in a range of neutron momenta of 1.4-5.2 GeV/c [16-18].

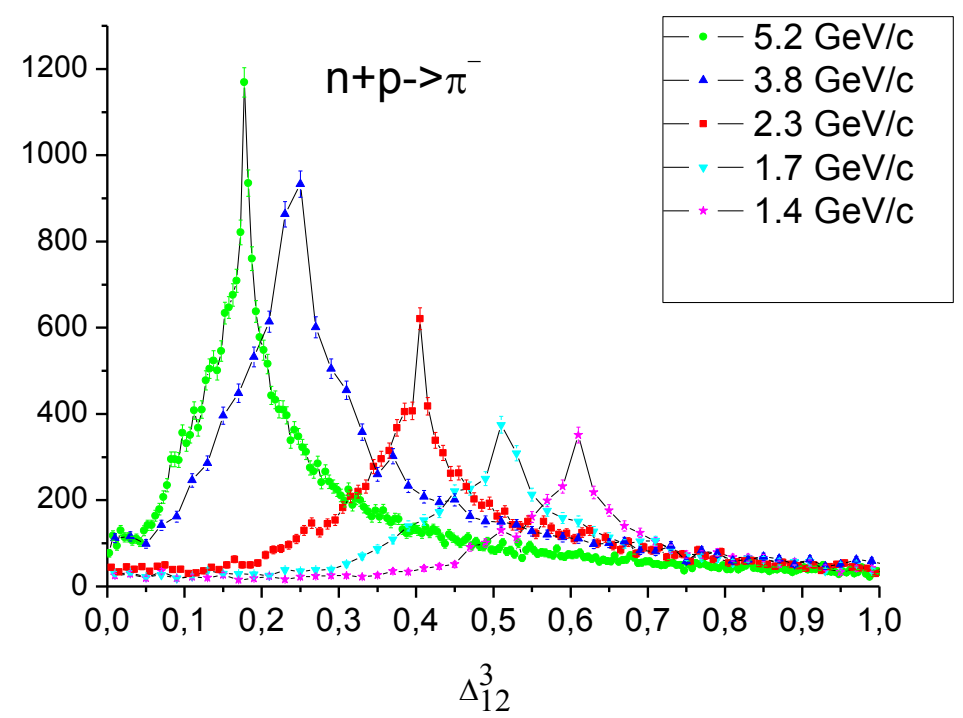

Figure 5. Pion production in $n+p$ reaction for different neutron momenta

Thus, the connection between the basic concept of the Lobachevsky geometry, the angle of parallelism, and the experimentally observed directed nuclear radiation, is proved. 
The new variable $\Delta_{12}^{3}$ provides a new and efficient criterion for analysis and selection of configurations and correlations in multiparticle production.

We give the following quotation to conclude the paper: "The standard model in elementary particle physics claims to be capable of describing electroweak and strong interactions and is a great achievement of experimental and theoretical physics of the second half of the 20th century. However, the standard model contains only those defining axioms which concern the Lagrangian symmetries, but this is not enough for the description of physical processes. Some additional conditions (hypotheses) are needed: initial and boundary conditions, assumptions about the constants entering the Lagrangians (masses, charges and so on)." [1].

Taking into account that, according to the criteria proposed by A. M. Baldin, the relativistic physics begins at rather moderate energies (beginning from $b_{i k} \sim 1$ ) (Figs. 2 , 3 ), the NICA collider under construction at LHEP JINR provides a great discovery potential, especially for investigation of fundamental laws (regularities) which cannot be deduced from the field theory (or the standard model).

\section{References}

1. A. M. Baldin, Selected papers devoted to Baldin's 70th birthday (JINR, Dubna, 1996)

2. W. Heisenberg, Phys. Today 29(3), 32-39 (1976)

3. R. Hofstadter, F. Bumiller, and M. R. Yearian, Rev. Mod. Phys. 30, 482 (1958)

4. R. P. Feynman, High Energy Collisions: Third International Conference at Stony Brook (N.Y. Gordon \& Breach, 1969) 237-249

5. J. Bjorken and E. Paschos, Phys. Rev. 185 (5), 1975-1982 (1969)

6. A.M. Baldin, Nucl. Phys. 18, 310 (1960)

7. A. Akhiezer and M. Rekalo, Biography of elementary particles (Naukova Dumka, Kiev, 1979)

8. A. M. Baldin, Dokl. Akad. Nauk 222(5), 1064 (1975)

9. PDG K. Hagiwara et al., Phys. Rev. D 66, 010001 (2002). (http://www-pdg.lbl.gov/)

10. A. A. Baldin, E. G. Baldina, E. N. Kladnitskaya, O. V. Rogachevsky, Phys. Part. Nucl. Lett. 1, 4(121), 7-16 (2004)

11. K. Hagiwara et al., Phys. Rev. D 66, 010001 (2002). (http://www-pdg.lbl.gov/)

12. N. A. Chernikov, Phys. Part. Nucl. 23(5), 1187 (1992)

13. A. P. Kotel'nikov, in Collected Papers "In Memoriam N. I. Lobatschevskij" V.2 (Kazan, 1927) 37-64

14. A. A. Baldin, Proc. International Seminar "Application and Development of Ideas of Lobachevsky in Modern Physics" (25-27 February 2004, Dubna, Russia, 2004) 103116

15. A. A. Baldin, in Proc. the XIX International Baldin Seminar on High Energy Physics Problems (ISHEPP XIX), Ed.: A.N. Sissakian, V.V. Burov, A.I. Malakhov, S.G. Bondarenko, E.B. Plekhanov, vol. 1 (Dubna, Sept.29 - Oct.4, 2008) 80-89

16. A. P.Gasparian et al., JINR Preprint 109111 (Dubna 1975)

17. A. P. Gasparian et al., Fiz. Tekhn. Eksper. 2, 37 (1977)

18. Yu. A. Troyan et al., Yad.Fiz. 63(9), 156 (2000) 